TITLE:

\title{
Shoulder horizontal abduction stretching effectively increases shear elastic modulus of pectoralis minor muscle
}

\section{AUTHOR(S):}

Umehara, Jun; Nakamura, Masatoshi; Fujita, Kosuke; Kusano, Ken; Nishishita, Satoru; Araki, Kojiro; Tanaka, Hiroki; Yanase, Ko; Ichihashi, Noriaki

\section{CITATION:}

Umehara, Jun ...[et al]. Shoulder horizontal abduction stretching effectively increases shear elastic modulus of pectoralis minor muscle. Journal of Shoulder and Elbow Surgery 2017, 26(7): 1159-1165

ISSUE DATE:

2017-07

URL:

http://hdl.handle.net/2433/231253

\section{RIGHT:}

(c) 2017. This manuscript version is made available under the CC-BY-NC-ND 4.0 license

http://creativecommons.org/licenses/by-nc-nd/4.0/; The full-text file will be made open to the public on 01 July 2018 in accordance with publisher's 'Terms and Conditions for Self-Archiving'; この論文は出版社版でありません。引用の際に は出版社版をご確認ご利用ください。; This is not the published version. Please cite only the published version. 
How do you stretch pectoralis minor?

1 Shoulder horizontal abduction stretching effectively increases shear elastic modulus of

2 pectoralis minor muscle

3

4 Jun Umehara, M.S. ${ }^{1,2)^{*}}$, Masatoshi Nakamura, Ph.D. ${ }^{3)}$, Kosuke Fujita, M.S. ${ }^{4)}$, Ken Kusano,

5 B.S. ${ }^{1)}$, Satoru Nishishita, M.S. ${ }^{1)}$, Kojiro Araki, M.S. ${ }^{5}$, Hiroki Tanaka, M.S. ${ }^{2)}$, Ko Yanase, M.S. ${ }^{1)}$,

6 Noriaki Ichihashi, Ph.D. ${ }^{1)}$

7

8 1) Human Health Sciences, Graduate School of Medicine, Kyoto University, Kyoto, Japan

9 2) Rehabilitation Unit, Kyoto University Hospital, Kyoto, Japan

10 3) Institute for Human Movement and Medical Sciences, Niigata University of Health and

11 Welfare, Niigata, Japan

12 4) Rehabilitation group, Department of Medical Technique, Nagoya University, Nagoya, Japan

13 5) Department of Rehabilitation, Sapporo Tokushukai Hospital, Sapporo, Japan

*Corresponding author

16 Jun Umehara $(\bowtie)$

17 Human Health Sciences, Graduate School of Medicine, Kyoto University,

1853 Shogoin-Kawahawa-cho, Kyoto 606-8507, Japan

19

Phone: +81-75-751-3935; Fax: +81-75-751-3909 
How do you stretch pectoralis minor?

20 E-mail: umehara.jun.77z@st.kyoto-u.ac.jp

21

22 Financial biases: None

23

24 Ethics committee approval

25 The study design was approved by the ethic committee of Kyoto University Graduate School

26 and the Faculty of Medicine (R0314).

27

28 Acknowledgement

29 This study was supported by the Grant - in - Aid for Scientific Research (B) $15 H 03043$.

30 
How do you stretch pectoralis minor?

\section{Abstract}

\section{Background}

The stretching maneuver for pectoralis minor muscle, which is shoulder horizontal abduction

or scapular retraction, is performed in a clinical and sport setting because the tightness of this

muscle may contribute to scapular dyskinesis. The effective stretching maneuver for pectoralis

minor muscle is unclear in vivo. The purpose of this study was to verify the effective stretching

maneuver for pectoralis minor muscle in vivo using ultrasonic shear wave elastography.

\section{Methods}

Eighteen healthy men participated in this study. Elongation of the pectoralis minor muscle was

measured for three stretching maneuvers (shoulder flexion, shoulder horizontal abduction, and scapular retraction) at three shoulder elevation angles $\left(30^{\circ}, 90^{\circ}\right.$, and $\left.150^{\circ}\right)$. The shear elastic modulus used as the index of muscle elongation was computed using ultrasonic shear wave elastography for the above nine stretching maneuver-angle combinations.

\section{Results}

The shear elastic modulus was highest in horizontal abduction $150^{\circ}$ followed by horizontal abduction $90^{\circ}$, horizontal abduction $30^{\circ}$, scapular retraction $30^{\circ}$, scapular retraction $90^{\circ}$, scapular retraction $150^{\circ}$, flex $150^{\circ}$, flex $90^{\circ}$ and flex $30^{\circ}$. The shear elastic modulus of horizontal abduction $90^{\circ}$ and horizontal abduction $150^{\circ}$ were significantly higher than other stretching 
How do you stretch pectoralis minor?

\section{Conclusions}

52 This study determined that shoulder horizontal abduction at elevations of $90^{\circ}$ and $150^{\circ}$ were

53 the most effective stretching maneuvers for pectoralis minor muscle in vivo.

54

55 Level of evidence: Basic Science Study.

56

57 Keyword

58 Stretching;

59 Pectoralis minor muscle;

60 Ultrasonic shear wave elastography;

61 Shear elastic modulus;

62 Shoulder horizontal abduction;

63 Rehabilitation

64 
How do you stretch pectoralis minor?

65

66

67

68

69

70

71

72

73

74

\section{Introduction}

In shoulder rehabilitation, clinical evaluation, and intervention for scapular dyskinesis are important because of its relation to various shoulder injuries, such as subacromial impingement ${ }^{4,12,17,19,23}$, rotator cuff tear ${ }^{14,24}$, unstable shoulder ${ }^{20}$ and frozen shoulder ${ }^{10}$. The literature suggests that scapular dyskinesis may be caused by multiple factors such as bone, joint, neurological, or soft tissue mechanisms ${ }^{13}$. In soft tissue mechanisms, the tightness of the pectoralis minor muscle (PMi) is one of the factors inducing scapular dyskinesis, which can be examined and treated by a therapist ${ }^{8}$. Previous studies have reported that the tightness of the PMi is related to posture, including scapular internal rotation in the resting position ${ }^{3}$ and decreases in scapular external rotation and posterior tilt during arm elevation ${ }^{5}$. These changes, which is the scapular internal rotation and anterior tilt, are similar to the change in scapular motion found in many shoulder injuries ${ }^{19,20}$ and it is also possible that there might be a relationship between shoulder injury and the tightness of the PMi. Therefore, the flexibility of the PMi is important for preventing and improving scapular dyskinesis.

Stretching interventions are recommended to increase and improve muscle flexibility and stretching of the PMi is frequently used in rehabilitation programs ${ }^{1,18,21}$. Therefore, some studies have investigated stretching maneuver of the PMi. In a previous study, Borstad and Ludewig $^{6}$ compared the length of the PMi during three stretching maneuver using an electromagnetic motion capture system with skin surface markers in healthy adults. This study concluded that the most effective PMi stretching maneuver was a unilateral corner self-stretch 
How do you stretch pectoralis minor?

85 similar to horizontal abduction of shoulder joint. On the other hand, Muraki et al. ${ }^{25}$ directly

86 measured the length of the PMi during three passive shoulder motions and three stretching

87 techniques using displacement sensors in fresh cadavers. They advocated that scapular

88 retraction made the greatest change in PMi length. The contradiction of these two studies most

89 likely resulted from differences between the subjects (living body vs. cadaver) or measurement

90 methods. In addition, it is unknown whether or not the results of these previous studies apply

91 to live people for effective stretching positions of PMi, because Borstad and Ludewig ${ }^{6}$ did not

92 measure the tension of the PMi during stretching, but instead measured the distance between

93 the coracoid process and the fourth rib/sternum junction, and Muraki et al. ${ }^{25}$ used cadavers in

94 their study. Therefore, an investigation of effective in vivo stretching maneuver of the PMi

95 determined by measuring muscle tension during stretching is needed.

A new ultrasound-based technology called ultrasonic shear wave elastography (SWE)

was developed, allowing reliable and non-invasive measurement of soft tissue viscoelastic

properties $^{2}$. SWE monitors the propagation of shear waves generated in tissue using acoustic

99 radiation forces and is able to evaluate the shear elastic modulus of individual muscles ${ }^{26}$.

100 Because of the strong liner relationship, identified by prior studies, between passive muscle

101 tension calculated by traditional method and shear elastic modulus measured by SWE in

102 vitro ${ }^{9,15}$, SWE has been used in many stretching studies of skeletal muscle ${ }^{9,15,27,28}$. In addition, 
How do you stretch pectoralis minor?

104 stretching ${ }^{27,28}$. Therefore, SWE has proven to be a valid technology for noninvasively

105 investigating muscle elongation in vivo.

106 Regarding stretching maneuver of the PMi, a unilateral corner self-stretch or scapular

107 retraction at a $30^{\circ}$ shoulder flexion angle have been recommended by Borstad and Ludewig ${ }^{6}$ or

108 Muraki et al. ${ }^{25}$, respectively. Muraki et al. ${ }^{25}$ also described that the PMi can be stretched by

$109150^{\circ}$ passive shoulder flexion and scaption as well as scapular retraction or shoulder horizontal

110 abduction. Thus, we hypothesized that shoulder horizontal abduction or scapular retraction with

111 the shoulder in elevated positions is an effective maneuver for stretching the PMi. The objective

112 of this study was to quantitatively verify the effective stretching maneuver of the PMi using the

113 shear elastic modulus measured by SWE in vivo. 
How do you stretch pectoralis minor?

\section{Materials and Methods}

\section{Participants}

117 Eighteen men (age, $26.2 \pm 4.0$ years; height, $171.1 \pm 5.0 \mathrm{~cm}$; weight, $67.4 \pm 7.8 \mathrm{~kg}$ ) with no

118 orthopaedic or nervous system abnormalities in their upper limbs participated in this study.

119 Participants were recruited from the students of our institution. The subject orally confirmed

120 that they complied with the following exclusion criteria: females, athletes, or those who perform

121 any extensive exercise, and subjects having a history of orthopaedic disease or neuropathy in

122 their upper limbs. The sample size was calculated by the $G^{*}$ power software (Version 3.1.,

123 Heinrich Heine University, Dusseldorf, Germany) for a one-way analysis of variance (ANOVA)

124 with repeated measures (effect size $=0.25, \alpha$ error $=0.05$, power $=0.8$ ), which showed that 17

125 subjects were required. This study protocol conformed to the Helsinki Declaration and was

126 approved by the Ethics Review Board of our institution.

\section{Experimental procedures}

129 This study was an experimental study, with randomized allocation of stretching intervention for

130 each subject using a random number table. Healthy male subjects were randomly recruited.

131 After the aim and procedures were explained to all subjects, the subjects underwent nine

132 stretching maneuvers performed by one researcher. The outcome was measured and analyzed

133 by another researcher. 
How do you stretch pectoralis minor?

135 therapist licenses: one investigator measured the shear elastic modulus using SWE, while the

136 other performed the stretching maneuver. The non-dominant upper limb was chosen for

137 intervention. Each subject lay on their side on a bed with their non-intervention arm under their

138 head, their trunk parallel to the long axis of the bed, and both the hip and knee flexed $45^{\circ}$. The

139 relax position (Rest) was defined as that the shoulder in $0^{\circ}$ flexion, $0^{\circ}$ abduction, the elbow

140 fully extended, and the palm of the hand parallel the bed. In this study, three stretching

141 maneuvers (flexion, horizontal abduction, and scapular retraction) were investigated at the three

142 shoulder elevation angles $\left(30^{\circ}, 90^{\circ}\right.$, and $\left.150^{\circ}\right)$ for a total of nine stretching positions, on the

143 basis of previous studies ${ }^{6,25}$. For passive shoulder motion, the interventional shoulder of the

144 subject was passively flexed at $30^{\circ}$ (Flex30), $90^{\circ}$ (Flex90), and $150^{\circ}$ (Flex150) by the

145 investigator (Figure 1). For shoulder horizontal abduction, the interventional shoulder was

146 passively horizontally abducted as much as possible at shoulder elevation angles of $30^{\circ}$ (Hab30),

$14790^{\circ}$ (Hab90), and $150^{\circ}$ (Hab150) while the shoulder was maximally externally rotated and the

148 elbow was flexed $90^{\circ}$ by the investigator (Figure 2). For scapular retraction, the interventional

149 fully flexed elbow was maximally pressed along the longitudinal axis of the humerus, and the

150 interventional scapular was passively maximally retracted at the shoulder for flexion angles of

$15130^{\circ}$ (Retract30), 90 (Retract90), and $150^{\circ}$ (Retraction150) by the investigator (Figure 3). The

152 subjects were stretched until reaching a point of discomfort (but not pain), as verbally

153 acknowledged by the subjects. During all stretching and measurement acquisition, subjects 
How do you stretch pectoralis minor?

154 were instructed to relax as much as possible.

\section{Instrumentation}

157 In this study, the shear elastic modulus measured by SWE (Aixplorer, SuperSonic Imagine,

158 Aix-en-Provence, France) with an ultrasound transducer (15-4 MHz linear probe) was defined

159 as the indicator of muscle elongation of the PMi. The shear elastic modulus was calculated from

160 the shear wave propagation speed generated by the transducer ${ }^{2}$. The shear elastic modulus (G)

161 was calculated from the shear wave propagation speed (V) using the following formula:

$162 \mathrm{G}=\rho \mathrm{V}^{2}$

163 Where $\rho$ is the muscle density, assumed to be $1,000 \mathrm{~kg} / \mathrm{m}^{3}$. A previous study showed that there

164 is a significant correlation between the shear elastic modulus, which was measured by SWE,

165 and the muscle elongation, which was measured by a traditional tension test ${ }^{9,15}$.

measurement place was defined as the midpoint between the coracoid process and the fourth

of the muscle belly in the ultrasound image. The shear elastic modulus was measured three

171 times at each measurement site and the mean value was used for analysis. All analyses were

172 performed by the researcher who was blinded to the stretching positions by anonymizing all 
How do you stretch pectoralis minor?

173 ultrasonic images. The subjects were instructed to hold their breath during measurement of the

174 shear elastic modulus to prevent PMi elongation due to the movement of the rib cage.

were used to evaluate the reliability of the ultrasound measurement. The measurement was

177 acquired for three passive shoulder motions and three stretching maneuvers. The reliability of

178 the shear elastic modulus measurement was confirmed using intraclass correlation coefficients

$179(1,3)\left(\mathrm{ICC}_{1,3}\right)$ with a 95\% confidence interval (95\% CI). ICC $_{1,3}$ values, which represent intra-

180 observer reliability in the intra-day, were calculated from the shear elastic modulus. ICC $\mathrm{I}_{1,3}$

181 values fell within a range of $0.90-0.99$ for all measurements (Table I). A previous study that

182 investigated the reliability coefficient suggested that a range of $0.81-1.00$ was almost perfect ${ }^{16}$.

183 Therefore, the measured values of the shear elastic modulus in our study were considered

184 reproducible since the ICC $_{1,3}$ observed was almost perfect, according to this previous study.

185

186 Data analysis

187 Statistical analysis was performed using IBM SPSS Statistics 22 (International Business

188 Machines Corporation). To find whether the PMi was elongated in the nine stretching positions,

189 differences in the shear elastic modulus between Rest and each stretching position were

190 assessed using a paired Student’s t-test with Bonferroni revision. Additionally, when the shear

191 elastic modulus was found to be significantly different from that at Rest, a one-way ANOVA 
How do you stretch pectoralis minor?

192 with repeated measures was used to determine the effect of passive motion or stretching

193 maneuver on the shear elastic moduli amongst them. If a significant main effect was found,

194 then a Bonferroni multiple comparison for the post hoc test was performed. A confidence level

195 of 0.05 was used in all statistical tests.

196 
How do you stretch pectoralis minor?

197

198

199

200

201

202

203 that at Rest, a one-way ANOVA with repeated measures was used to indicate a significant main

204 effect $(\mathrm{P}<.001, \mathrm{~F}=29.0)$. For the positions showing significantly higher shear elastic moduli

205 than the elastic modulus at Rest, a Bonferroni multiple comparison for the post-hoc test was

206 performed, indicating that the shear elastic moduli of Hab90 and Hab150 were significantly

207 higher than those of the other positions. However, there was no significant difference between

208 Hab90 and Hab150. In addition, although the shear elastic modulus of Hab30 was significantly

209 higher than those of Flex150 and Retraction150 ( $<.001$, respectively), there were no

210 significant differences among the others (Figure 4).

211 
How do you stretch pectoralis minor?

\section{Discussion}

213 This is the first to determine effective stretching maneuver of the PMi using shear elastic

214 modulus values measured by SWE, which quantitatively reflects the grade of muscle elongation

215 during stretching in vivo. The main finding of this study was that maximal horizontal abduction

216 of the shoulder at an elevation angle of $90^{\circ}$ and $150^{\circ}$ effectively elongates the PMi muscle.

We hypothesized that the PMi could be elongated effectively by shoulder horizontal

218 abduction or scapular retraction at elevated shoulder positions i.e., Hab150 or Retraction150.

219 In this study, our results showed that the shear elastic modulus at all measurement positions

220 was higher than that at Rest, except for Flex30 and Flex90. Furthermore, the shear elastic

221 moduli of Hab90 and Hab150 were significantly greater than those of all measurement positions

222 whose shear elastic modulus was greater than at Rest. These results suggest that the most

223 effective stretching maneuvers of the PMi are Hab90 and Hab150, which is partly consistent

224 with our hypothesis.

Borstad and Ludewig ${ }^{6}$ compared the mean length change from the coracoid process of

226 the scapula to the fourth rib/sternum junction for three pectoralis minor stretches; unilateral

227 corner self-stretch, sitting manual stretch, and spine manual stretch. They concluded that

228 unilateral corner self-stretch, in which a subject abducts the humerus to $90^{\circ}$ with the palm on a

229 wall and then rotates their trunk away from the elevated arm to increase the shoulder horizontal

230 abduction, lengthened the pectoralis minor muscle most effectively. Our results, that the shear

231 elastic moduli of Hab90 and Hab150 were significantly higher than those of other measurement 
How do you stretch pectoralis minor?

232 positions, were consistent with the findings of the previous study by Borstad and Ludewig ${ }^{6}$. On

233 the other hand, Muraki et al. directly measured PMi lengthening during three passive shoulder

234 motions and three stretching techniques using fresh cadaveric transthoracic specimens. Their

235 study concluded that scapular retraction at an angle of $30^{\circ}$ flexion, in which the examiner

236 exerted a posterosuperior pressure to the elbow along the longitudinal axis of the humerus,

237 resulted in the greatest change in PMi length measured by displacement sensor ${ }^{25}$, this is

238 inconsistent with our results. This contradiction probably originates from the difference in

239 methodology. The horizontal abduction of the shoulder might stretch the pectoralis major

240 muscle and the clavipectoral fascia, which may directly impact the ability of the pectoralis

241 minor muscle to receive stretch influence. Removing these tissues overlying the PMi in order

242 to expose this muscle, as stated in Murakis' study, could be the reason for the contradiction. In

243 addition, there was a glaring difference in the nature of the study medium, i.e., live tissue versus

244 cadaver tissue, which likely contributed to this inconsistency. It is possible that the difference

245 in viscoelasticity and other material properties of the shoulder joint between a living body and

246 a cadaver affected the elongation of the $\mathrm{PMi}^{11}$. Contrary to these previous studies, our study

247 examined the applicability of various stretching maneuver of the PMi in live people using the

248 shear elastic modulus values measured by SWE. the elastic modulus of Flex150. These results indicate that shoulder horizontal abduction is a 
How do you stretch pectoralis minor?

251 more effective means of stretching the PMi than shoulder flexion, and that scapular motion is

252 probably responsible for this difference. From an anatomical perspective, the external rotation

253 and posterior tilt of the scapula stretches the $\mathrm{PMi}^{6}$ because this muscle originates on the third,

254 fourth, and fifth ribs and runs superolaterally, inserting at the coracoid process of the scapula.

255 Previous studies measuring scapular motion reported that during shoulder flexion the scapula

256 externally rotates, upwardly rotate, and tilts posteriorly ${ }^{22}$, and that the scapula externally rotates

257 and tilts posteriorly during shoulder horizontal abduction ${ }^{7}$. Comparing the scapular motion of

258 shoulder flexion and that of shoulder horizontal abduction in these previous studies, the scapular

259 external rotation during shoulder horizontal abduction was greater than that during shoulder

260 flexion. Thus, the results of the current study indicating that the shear elastic moduli of Hab30,

261 Hab90, and Hab150 were significantly higher than the elastic modulus of Flex150 suggests that

262 scapular external rotation contributes more to stretching the PMi than scapular posterior tilt.

263 Furthermore, the scapular motion also relates to the fact that the shear elastic moduli of Hab90

264 and Hab150 were found to be significantly higher than those of Retraction30, Retraction90, and

265 Retraction150. The PMi could be more stretched by shoulder horizontal abduction than scapular

266 retraction because the scapular external rotation of shoulder horizontal abduction is greater than

267 that of scapular retraction. However, there was no study investigating scapular motion during

268 scapular retraction. Further research is required to elucidate scapular motion during scapular

retraction using electromagnetic sensors or optoelectronic markers. 
How do you stretch pectoralis minor?

measurement positions, it was found to be significantly lower than all except the Flex30 and

272 Flex90 positions. Considering these results, although Hab90 and Hab150 were the most

273 effective for stretching the PMi, all measurement positions, except Flex30 and Flex90,

274 effectively stretch the PMi. In the clinical setting, patients requiring stretching of the PMi

275 frequently have a limited range of shoulder motion, and a shoulder instability. Therefore, Hab30

276 or Retraction30 might be better suited for these patients. Further research is required to

277 investigate the effects of stretching intervention of the PMi in the patients with a limited range

278 of motion and a shoulder instability.

Our determination of horizontal abduction of the shoulder at elevation angles of $90^{\circ}$

280 and $150^{\circ}$ as effective means of stretching the PMi may be beneficial in clinical and athletic

281 settings. However, this study should be interpreted with note of the following. First, the

282 participants in this study were healthy young men as prescribed by the exclusion criteria.

283 Therefore, it is unknown whether similar effects can always be expected in patients with

284 impingement syndrome or frozen shoulder. Second, we could not measure the scapular motion

285 during the stretching maneuvers. Further research investigating scapular motion during

286 stretching is required to identify any potential relationship between scapular motion and

287 elongation of the PMi. Third, the shear elastic modulus of the lateral fiber groups of the PMi

288 was measured in this study; thus, similar behavior cannot always be expected in the medial 
How do you stretch pectoralis minor?

289 fiber groups of the PMi. However, we presume that there are few differences between the shear

290 elastic moduli of the lateral and medial fiber groups of the PMi because Muraki et al. ${ }^{25}$ reported

291 that there was no difference of lengthening of the PMi. Thus, the shear elastic modulus of the

292 PMi measured in the current study might represent that of the whole PMi muscle.

293 
How do you stretch pectoralis minor?

\section{Conclusions}

295 We quantitatively investigated effective stretching maneuver of the pectoralis minor muscle

296 using shear elastic modulus values obtained by ultrasonic shear wave elastography. Our results

297 showed that shoulder horizontal abduction at shoulder elevation angles of $90^{\circ}$ and $150^{\circ}$

298 effectively elongated the pectoralis minor muscle. The stretching maneuver of the pectoralis

299 minor muscle proposed in this study may be useful for clinical application.

300 
How do you stretch pectoralis minor?

301

302

303

304

305

306

307

308

309

310

311

312

313

314

315

316

317

318

\section{Reference}

1. Bang MD, Deyle GD. Comparison of supervised exercise with and without manual physical therapy for patients with shoulder impingement syndrome. J. Orthop. Sports Phys. Ther. 2000 Mar;30(3):126-37. doi:10.2519/jospt.2000.30.3.126

2. Bercoff J, Tanter M, Fink M. Supersonic shear imaging: A new technique for soft tissue elasticity mapping. IEEE Trans. Ultrason. Ferroelectr. Freq. 2004 Apr;51(4):396-409. doi:10.1109/TUFFC.2004.1295425

3. Borstad JD. Resting position variables at the shoulder: evidence to support a postureimpairment association. Phys. Ther. 2006 Apr;86(4):549-57.

4. Borstad JD, Ludewig PM. Comparison of scapular kinematics between elevation and lowering of the arm in the scapular plane. Clin. Biomech. 2002;17:650-659. doi:10.1016/S0268-0033(02)00136-5

5. Borstad JD, Ludewig PM. The effect of long versus short pectoralis minor resting length on scapular kinematics in healthy individuals. J. Orthop. Sports Phys. Ther. 2005;35(4):227-238. doi:10.2519/jospt.2005.1669

6. Borstad JD, Ludewig PM. Comparison of three stretches for the pectoralis minor muscle. J. Shoulder Elbow Surg. 2006 Jan;15(3):324-30. doi:10.1016/j.jse.2005.08.011

7. Bourne D a., Choo AMT, Regan WD, MacIntyre DL, Oxland TR. Three-dimensional rotation of the scapula during functional movements: An in vivo study in healthy volunteers. J. Shoulder Elb. Surg. 2007;16(2):150-162. doi:10.1016/j.jse.2006.06.011 
How do you stretch pectoralis minor?

321 8. Cools AMJ, Struyf F, De Mey K, Maenhout A, Castelein B, Cagnie B. Rehabilitation of scapular dyskinesis: from the office worker to the elite overhead athlete. Br. J.

323 Sports Med. 2014;48(8):692-7. doi:10.1136/bjsports-2013-092148

324

9. $\quad$ Eby SF, Song P, Chen S, Chen Q, Greenleaf JF, An K-N. Validation of shear wave 325 elastography in skeletal muscle. J. Biomech. 2013 Sep 27;46(14):2381-7. doi:10.1016/j.jbiomech.2013.07.033

10. Fayad F, Roby-Brami A, Yazbeck C, Hanneton S, Lefevre-Colau M-M, Gautheron V, et al. Three-dimensional scapular kinematics and scapulohumeral rhythm in patients with glenohumeral osteoarthritis or frozen shoulder. J. Biomech. 2008;41(2):326-332. doi:10.1016/j.jbiomech.2007.09.004

331 11. Gottsauner-Wolf F, Grabowski JJ, Chao EYS, An KN. Effects of freeze/thaw conditioning on the tensile properties and failure mode of bone-muscle-bone units: A biomechanical and histological study in dogs. J. Orthop. Res. 1995;13(1):90-95. doi:10.1002/jor.1100130114

12. Hébert LJ, Moffet H, McFadyen BJ, Dionne CE. Scapular behavior in shoulder impingement syndrome. Arch. Phys. Med. Rehabil. [Internet]. 2002;83(1):60-69. doi:10.1053/apmr.2002.27471

13. Kibler W Ben, Ludewig PM, McClure PW, Michener L a, Bak K, Sciascia AD. 
How do you stretch pectoralis minor?

340

341

342

344

345

346

statement from the “Scapular Summit”. Br. J. Sports Med. [Internet]. 2013;47(14):877-

85. doi:10.1136/bjsports-2013-092425

14. Kijima T, Matsuki K, Ochiai N, Yamaguchi T, Sasaki Y, Hashimoto E, et al. In vivo 3dimensional analysis of scapular and glenohumeral kinematics: comparison of symptomatic or asymptomatic shoulders with rotator cuff tears and healthy shoulders.

J. Shoulder Elb. Surg. 2015;24(11):1817-1826. doi:10.1016/j.jse.2015.06.003

15. Koo TK, Guo J-Y, Cohen JH, Parker KJ. Relationship between shear elastic modulus and passive muscle force: an ex-vivo study. J. Biomech. 2013 Aug 9;46(12):2053-9. doi:10.1016/j.jbiomech.2013.05.016

16. Landis JR, Koch GG. The measurement of observer agreement for categorical data. Biometrics. 1977 Mar;33(1):159-74.

17. Lawrence RL, Braman JP, Laprade RF, Ludewig PM. Comparison of 3-Dimensional Shoulder Complex Kinematics in Individuals With and Without Shoulder Pain, Part 1: Sternoclavicular, Acromioclavicular, and Scapulothoracic Joints. J. Orthop. Sport. Phys. Ther. 2014;44(9):636-A8. doi:10.2519/jospt.2014.5339

18. Ludewig PM, Borstad JD. Effects of a home exercise programme on shoulder pain and functional status in construction workers. Occup Envirom Med. 2003;60:841-849.

19. Ludewig PM, Cook TM. Alterations in shoulder kinematics and associated muscle activity in people with symptoms of shoulder impingement. Phys. Ther. 2000 
How do you stretch pectoralis minor?

359

360

361

362

363

364

365

366

367

368

369

370

371

372

373

374

375

Mar;80(3):276-91.

20. Matias R, Pascoal AG. The unstable shoulder in arm elevation: A three-dimensional and electromyographic study in subjects with glenohumeral instability. Clin. Biomech. 2006;21:S52-S58. doi:10.1016/j.clinbiomech.2005.09.014

21. McClure PW, Bialker J, Neff N, Williams G, Karduna A. Shoulder function and 3dimensional kinematics in people with shoulder impingement syndrome before and after a 6-week exercise program. Phys. Ther. 2004 Sep;84(9):832-48.

22. McClure PW, Michener L a., Sennett BJ, Karduna AR. Direct 3-dimensional measurement of scapular kinematics during dynamic movements in vivo. J. Shoulder Elb. Surg. 2001;10(3):269-277. doi:10.1067/mse.2001.112954

23. McClure PW, Michener L a, Karduna AR. Shoulder function and 3-dimensional scapular kinematics in people with and without shoulder impingement syndrome. Phys. Ther. 2006;86(8):1075-1090.

24. Mell AG, LaScalza S, Guffey P, Ray J, Maciejewski M, Carpenter JE, et al. Effect of rotator cuff pathology on shoulder rhythm. J. Shoulder Elb. Surg. 2005;14(1):S58-S64. doi:10.1016/j.jse.2004.09.018

25. Muraki T, Aoki M, Izumi T, Fujii M, Hidaka E, Miyamoto S. Lengthening of the Pectoralis Minor Muscle During Passive Shoulder Motions and Stretching Techniques: A Cadaveric Biomechanical Study. Phys. Ther. 2009 Apr 1;89(4):333-341. 
How do you stretch pectoralis minor?

378

379

380

381

382

383

384

385

386

387

388

389

390

391 doi:10.2522/ptj.20080248

26. Shiina T, Nightingale KR, Palmeri ML, Hall TJ, Bamber JC, Barr RG, et al. WFUMB Guidelines and Recommendations for Clinical Use of Ultrasound Elastography: Part 1: Basic Principles and Terminology. Ultrasound Med. Biol. 2015 May;41(5):1126-1147. doi:10.1016/j.ultrasmedbio.2015.03.009

27. Umegaki H, Ikezoe T, Nakamura M, Nishishita S, Kobayashi T, Fujita K, et al. The effect of hip rotation on shear elastic modulus of the medial and lateral hamstrings during stretching. Man. Ther. 2014 Aug 13;7-10. doi:10.1016/j.math.2014.07.016

28. Umehara J, Ikezoe T, Nishishita S, Nakamura M, Umegaki H, Kobayashi T, et al. Effect of hip and knee position on tensor fasciae latae elongation during stretching: An ultrasonic shear wave elastography study. Clin. Biomech. 2015 Sep;30(10):1056-1059. doi:10.1016/j.clinbiomech.2015.09.007 
How do you stretch pectoralis minor?

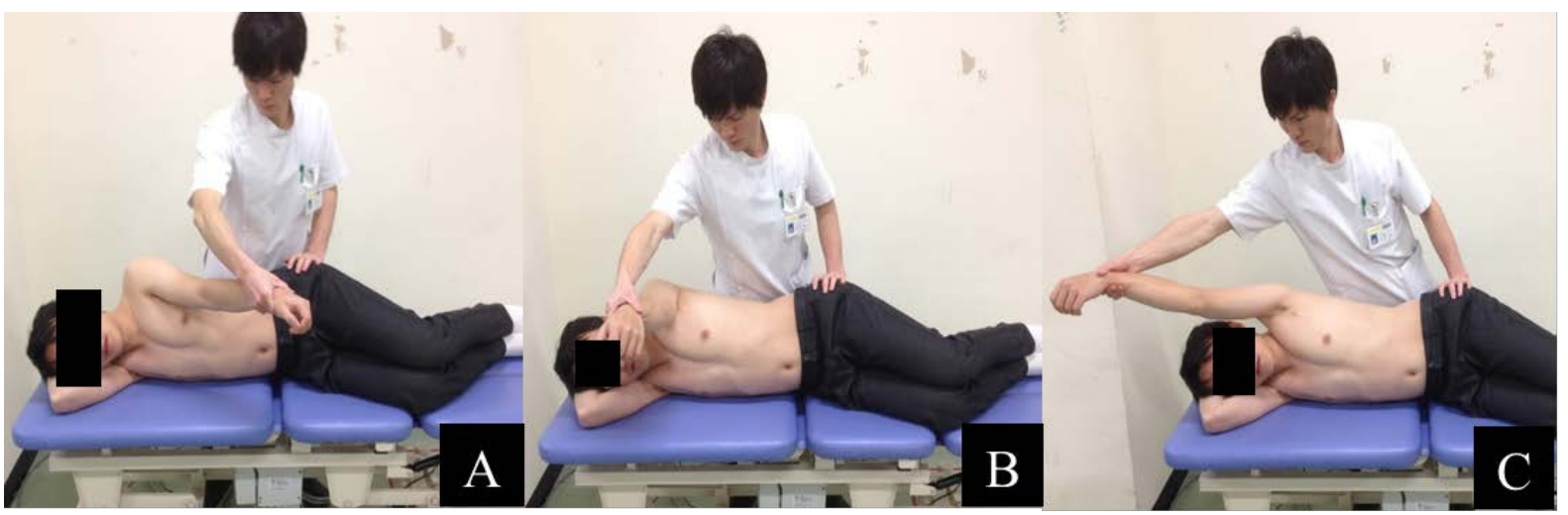

392 Figure 1. Passive shoulder flexion; left figure is Flex30, centre figure is Flex90, and right figure 393 is Flex150.

394 
How do you stretch pectoralis minor?

395

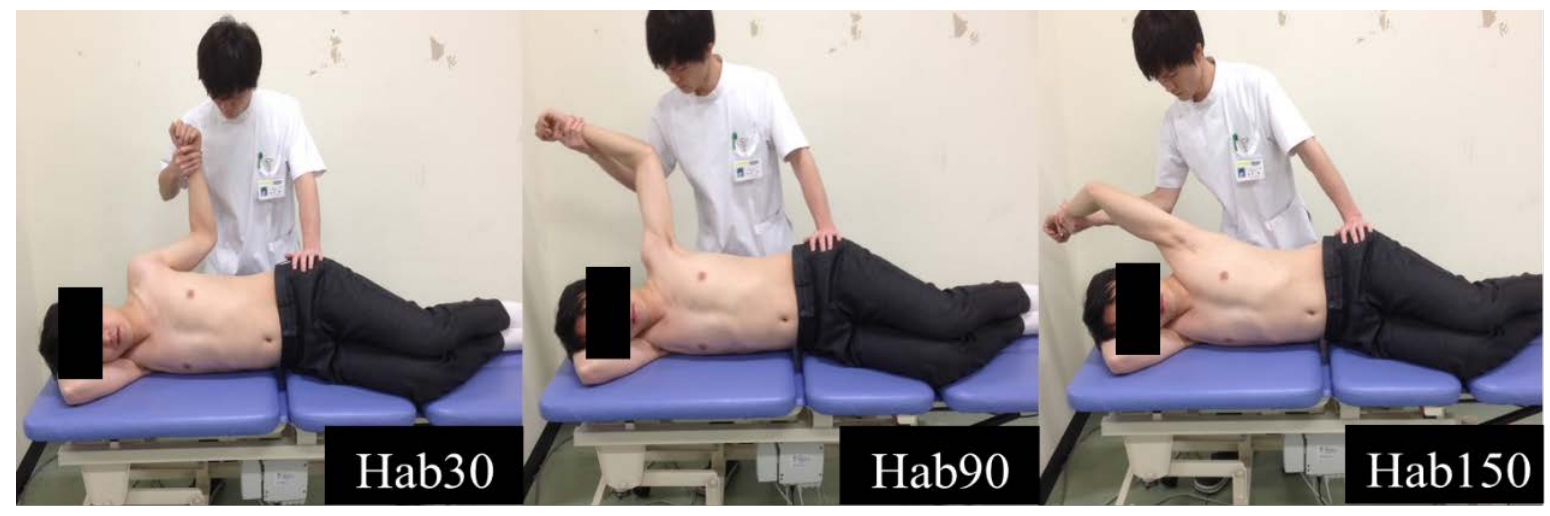

396 Figure 2. Shoulder horizontal abduction stretching; A is Hab30, B is Hab90, and C is Hab150. 
How do you stretch pectoralis minor?

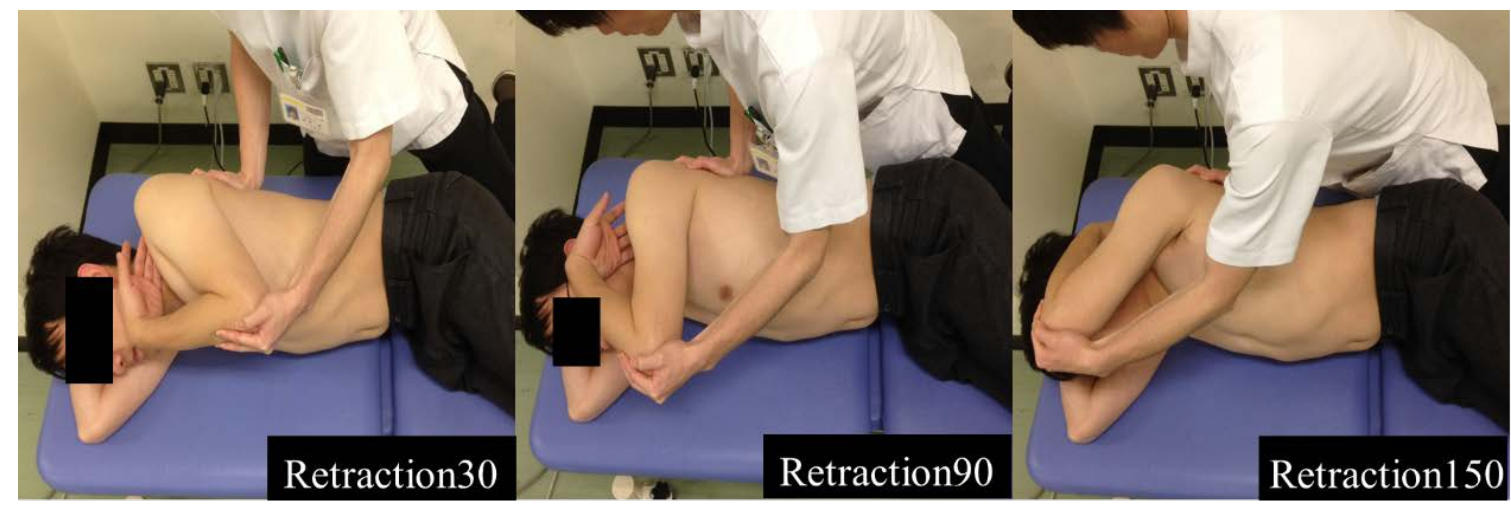

399 Figure 3. Scapular retraction stretching; A is Retraction30, B is Retraction90, and C is 400 Retraction150.

401 
How do you stretch pectoralis minor?



403 Figure 4. Multiple comparisons of shear elastic modulus; *: p $<.01$ Hab150 is significantly

404 higher than others except for Hab90, †: $\mathrm{p}<.01$ Hab90 is significantly higher than others except 405 for Hab150, ¥: p<.001 Hab30 is significantly higher than Flex150 and Retraction150. 
How do you stretch pectoralis minor?

407 Table I Reliability of shear elastic modulus.

\begin{tabular}{ccc}
\hline Measurement position & ICC $_{1,3}$ & $95 \%$ CI \\
\hline Rest & 0.99 & $0.94-1.00$ \\
Flex30 & 0.95 & $0.79-1.00$ \\
Flex90 & 0.94 & $0.74-0.99$ \\
Flex150 & 0.97 & $0.86-1.00$ \\
Hab30 & 0.90 & $0.55-0.99$ \\
Hab90 & 0.95 & $0.77-0.99$ \\
Hab150 & 0.99 & $0.94-1.00$ \\
Retraction30 & 0.95 & $0.78-0.99$ \\
Retraction90 & 0.95 & $0.76-0.99$ \\
Retraction150 & 0.93 & $0.69-0.99$ \\
\hline
\end{tabular}

408 ICC: intraclass correlation coefficient, 95\% CI: 95\% confidence interval 
How do you stretch pectoralis minor?

410 Table II Shear elastic modulus of pectoralis minor muscle in measurement positions.

\begin{tabular}{cccc}
\hline Measurement position & $\begin{array}{c}\text { shear elastic modulus } \\
\text { mean value } \pm \text { SD }(\mathrm{kPa})\end{array}$ & 95\% CI & $\begin{array}{c}\text { Comparison with Rest } \\
\text { (P value) }\end{array}$ \\
\hline Rest & $12.5 \pm 2.6$ & $11.3-13.7$ & .99 \\
Flex30 & $12.8 \pm 4.3$ & $10.8-14.8$ & .54 \\
Flex90 & $10.3 \pm 3.0$ & $9.0-11.7$ & .02 \\
Flex150 & $18.0 \pm 5.8$ & $15.3-20.7$ & $<.001$ \\
Hab30 & $31.1 \pm 7.0$ & $27.9-34.4$ & $<.001$ \\
Hab90 & $56.7 \pm 19.1$ & $47.9-65.6$ & $<.001$ \\
Hab150 & $58.7 \pm 20.0$ & $49.5-68.0$ & .003 \\
Retraction30 & $27.8 \pm 13.4$ & $21.6-34.0$ & .007 \\
Retraction90 & $24.0 \pm 12.2$ & $18.4-29.7$ & .02 \\
Retraction150 & $19.3 \pm 7.7$ & $15.7-22.8$ & \\
\hline
\end{tabular}

411 SD: standard deviation, 95\% CI: 95\% confidence interval 